Received: 25 January 2017

Accepted: 5 April 2018

Published online: 25 April 2018

\section{Yucca fern shaped CuO nanowires on Cu foam for remitting capacity fading of Li-ion battery anodes}

\author{
Zhifeng Wang ${ }^{1,2}$, Yanshan Zhang ${ }^{1}$, Hanqing Xiong ${ }^{3}$, Chunling Qin ${ }^{1}{ }^{1}$, Weimin Zhao $^{1}$ \& \\ Xizheng Liv $\mathbb{D}^{2,4}$
}

To remit capacity fading of lithium ion battery (LIB) anodes, freestanding yucca fern shaped CuO nanowires (NWs) on Cu foams are fabricated as anodes by combining facile and scalable anodization of copper foams followed by calcination. The porous and radial configuration of the hierarchical CuO NWs on the Cu foam substrate guarantees the remarkably improved electrochemical performance with durable cycle stability and excellent rate capability compared with CuO NWs on Cu foils. The reversible capacity remains $461.5 \mathrm{mAh} / \mathrm{g}$ after 100 repeated cycles at a current density of $100 \mathrm{~mA} / \mathrm{g}$, and a capacity of $150.6 \mathrm{mAh} / \mathrm{g}$ even at a high rate of $1000 \mathrm{~mA} / \mathrm{g}$. By examining the surface morphology of the cycled samples, possible performance fading route is proposed. The 3D CuO NWs network with a porous architecture simutaneously reduces the ion diffusion distances, promotes the electrolyte permeation and electronic conductivity. This novel strategy might open a new window to develop durable CuO based composite anodes for LIBs.

The explosive growth of electronics market and electric vehicle industries raise stringent requirement for more reliable, cost-effective and high capacity energy storage devices. Lithium ion battery (LIB) is one of the dominant choices due to its advantages such as high energy density, high operation potential and long cycle life since their commercialization $^{1,2}$. However, the rapid market growth requires better batteries in terms of capacity, durability and cost, which depends on the key electrode materials. The currently used graphite anode prohibited a higher specific capacity and energy density due to its relatively lower theoretical capacity of $372 \mathrm{mAh} / \mathrm{g}^{3,4}$. Therefore, it is highly urgent to develop new anode materials with higher electrochemical performance and lower cost. Transition metal oxides $\left(\mathrm{M}_{x} \mathrm{O}_{y}\right.$ where $\mathrm{M}$ can be $\mathrm{Mn}, \mathrm{Fe}, \mathrm{Co}, \mathrm{Ni}, \mathrm{Cu}$ and so on) exhibit super Li ions storage capability $(600 \sim 1300 \mathrm{mAh} / \mathrm{g})$ through a conversion reaction mechanism, which make them promising candidates for next generation LIBs anodes ${ }^{5-7}$. Unfortunately, the conversion reaction processes encountered with huge volume variation, pulverization and disconnected from the current collector during discharge/charge cycles. Thus, metal oxide anodes have not yet been practical utilized. To conquer these issues, tremendous efforts are devoted to fabricating nanoscale materials such as nanoparticles, nanowires, nanorods, nanotubes, nanosheets and their electrochemical performance have been improved to some extent ${ }^{8}$. Another effective strategy is to integrating the transition metal oxides into conductive network such as carbon nanotubes, graphene and so on ${ }^{9-11}$. Most of the nanoscale metal oxides or corresponding composite are fabricated into electrode by blending with organic binders. However, the weak bonding power between commonly used binders and anode materials are usually failed to meet the electrode volume expansions. In addition, the insulation essence of organic binder deteriorates the electrochemical performance. Therefore, freestanding and binder-free novel structures and materials are in demand and being developed ${ }^{9-12}$.

Among various transition metal oxides, $\mathrm{CuO}$ is drawing great focus due to its merits such as high theoretical capacity of $674 \mathrm{mAh} / \mathrm{g}^{13}$, low cost, environmental benignity and ease of production. Nevertheless, similar with other transition metal oxides, it is still hindered by the morphological collapse caused by the volume fluctuation

\footnotetext{
${ }^{1}$ Key Laboratory for New Type of Functional Materials in Hebei Province, School of Materials Science and Engineering, Hebei University of Technology, Tianjin, 300130, China. ${ }^{2}$ Tianjin Key Laboratory of Advanced Functional Porous Materials, Institute for New Energy Materials and Low-Carbon Technology, School of Materials Science and Engineering, Tianjin University of Technology, Tianjin, 300384, China. ${ }^{3}$ School of Materials Science and Engineering, Central South University, Changsha, 410083, China. ${ }^{4}$ Key Laboratory of Advanced Energy Materials Chemistry (Ministry of Education), Nankai University, Tianjin, 300071, China. Correspondence and requests for materials should be addressed to C.Q. (email: clqin@hebut.edu.cn) orX.L. (email: xzliu@tjut.edu.cn)
} 


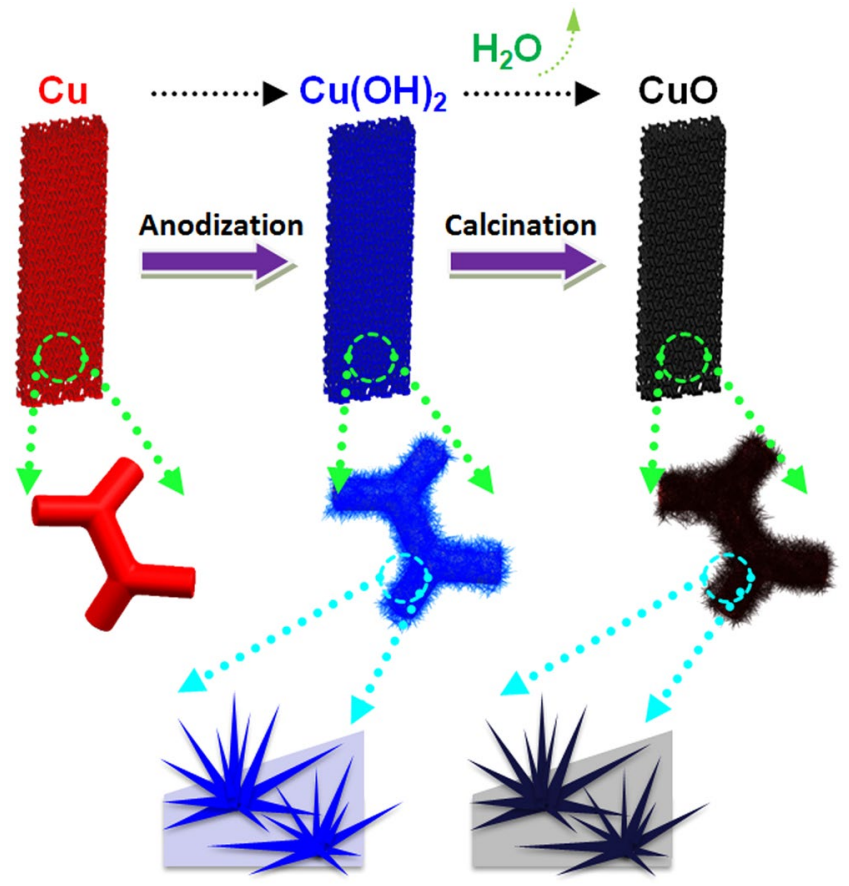

Figure 1. Schematic illustration of the fabrication process of hierarchical porous $\mathrm{CuO}$ composites.

along with the lithiation/delithiation ${ }^{13-16}$. Although the conductive $\mathrm{Cu}$ appeared as one of the important lithiation products, it is hardly connected together to form an electrons transport way to the current collector, causing a low conductivity for the electrode composite. Facing these challenges, it is necessary to adopt new architecture design and material preparation processes. The morphology controlled copper oxides (porous microspheres, flower-like, and thorn-like $\mathrm{CuO}$ ) were thoroughly investigated as anodes for LIBs. It is found that the $\mathrm{CuO}$ microspheres exhibit a superior cycle stability of $429.0 \mathrm{mAh} / \mathrm{g}$ after $50 \mathrm{cycles}^{17}$. Furthermore, design and fabrication of freestanding and binder-free electrode composite could improve the conductivity and accommodation ability of volume expansions, which have been proved to be an effective strategy to enhance the electrochemical performance. Yuan et al. prepared $\mathrm{CuO}$ nanoarrays by utilizing the ammonia solution to engrave the $\mathrm{Cu}$ foil as free standing anode ${ }^{18}$. Tan et al. fabricated freestanding cable-like $\mathrm{CuO} /$ carbon-nitride core-shell nanostructures which obviously improved energy/power densities of the whole electrode ${ }^{12}$. Chen et al. developed in-situ etched and oxidated the specified $\mathrm{Cu}$ foil method for preparation the 3D network architecture with flower-like nanosheets connected by nanowires, which exhibited better electrochemical performance as anode for rechargeable batteries ${ }^{19}$. We recognize that freestanding materials with porous structure offer great benefits to enhance the electrochemical properties. Thus, porous nanowires combined the $3 \mathrm{D}$ conductive network will have superior performance as anodes for LIBs.

Herein, we report on an elaborate design of freestanding yucca fern shaped CuO nanowires (NWs)@Cu foam with hierarchical porous structure through facile and scalable anodization method and their application as anodes for LIBs. For comparison, CuO NWs@Cu foil was also prepared by the same method. Owing to the unique yucca fern shaped $\mathrm{CuO}$ cluster and a synergistic effect with the 3D conductive network, this CuO NWs@ $\mathrm{Cu}$ foam composite demonstrates a better electrochemical performance compared to CuO NWs@Cu foil anode. The detailed mechanisms have been carefully examined by investigating the morphology evolution on the cycled composite electrodes. Due to the unique properties and facile preparation method, the CuO NWs@Cu foam composite displays promising applications as next-generation anode for LIBs.

\section{Results}

The typical preparation process of the 3D hierarchical porous $\mathrm{CuO}$ NWs on $\mathrm{Cu}$ foam is displayed in Fig. 1. The $\mathrm{Cu}(\mathrm{OH})_{2}$ NWs clusters were first obtained through the electrochemical anodization of Cu foam in an alkaline aqueous solution. The $\mathrm{Cu}$ atoms at the surface were oxidized to $\mathrm{Cu}^{2+}$ ions and immediately reacted with $\mathrm{OH}^{-}$ from the alkaline solution to form $\mathrm{Cu}(\mathrm{OH})_{2}$ and deposited on the surface. The thickness and morphology can be tuned by the optimizing electrolyte concentration, current density, reaction temperature and time ${ }^{20,21}$. During calcination, the dehydration reaction of $\mathrm{Cu}(\mathrm{OH})_{2}$ NWs occurs at high temperature. Plenty of vacancies and pores were generated at dehydration sites of $\mathrm{Cu}(\mathrm{OH})_{2}$. As a result, $\mathrm{CuO}$ NWs with porous structure are finally obtained. The digital photographs of the bare $\mathrm{Cu}$ foam, anodized foam and heat treated sample are shown in Fig. S1. The red copper foam changed to navy blue after anodization and then to brownish black after calcination, which are corresponding to the formation of $\mathrm{Cu}(\mathrm{OH})_{2}$ and $\mathrm{CuO}$ on the $\mathrm{Cu}$ foam substrates.

The surface morphology of the bare Cu foam and the as-obtained CuO NWs@Cu foam was characterized by SEM and TEM. As the substrate, $\mathrm{Cu}$ foam was first characterized by SEM as shown in Fig. 2a. A smooth surface 

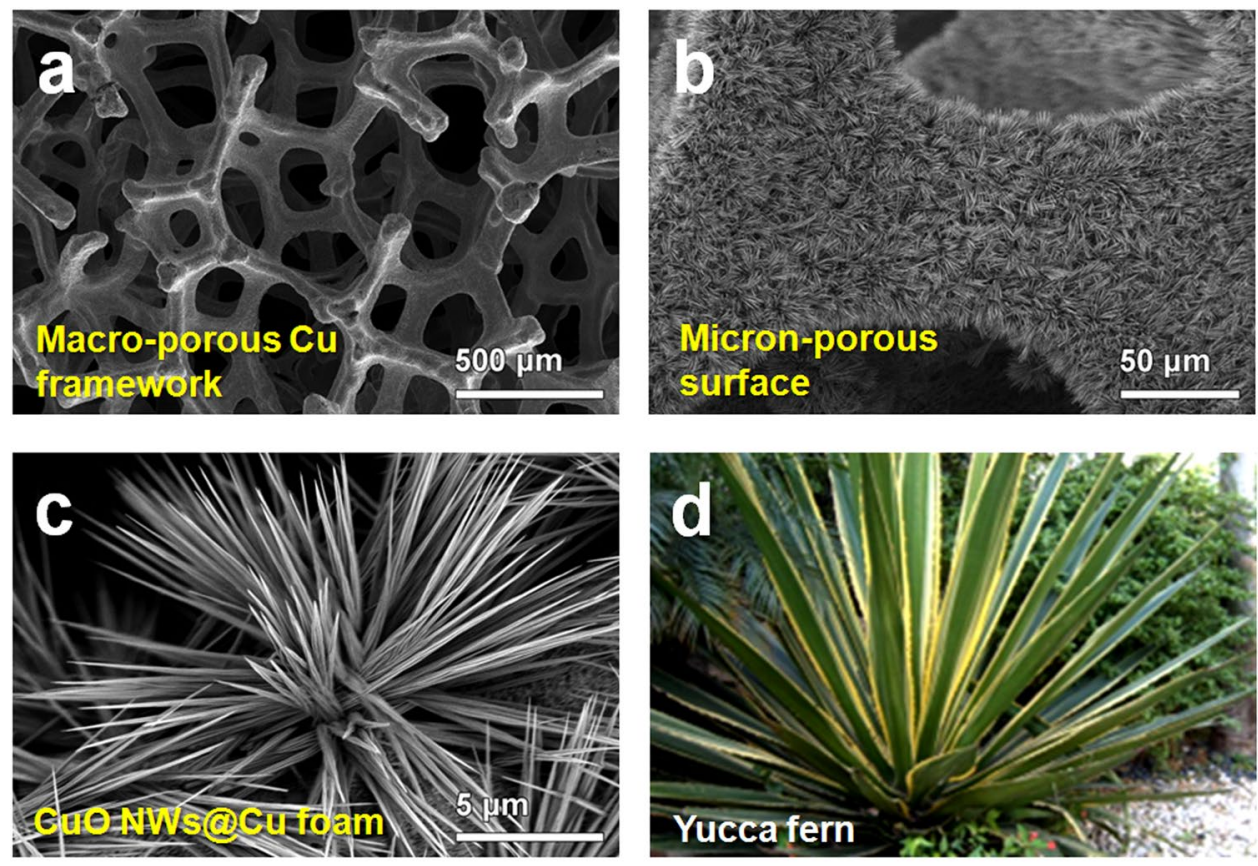

Figure 2. (a) SEM image of bare $\mathrm{Cu}$ foam, (b,c) Low- and high-magnification SEM images of CuO NWs grown on $\mathrm{Cu}$ foam, (d) Digital photograph of Yucca fern.

and 3D interconnected network with 50-80 micrometers ligament size and 200-500 micrometers pore size has been confirmed. After the anodization and calcination, uniform $\mathrm{CuO}$ NWs with a high density have been fabricated as displayed in Fig. 2b. A higher magnification image of $\mathrm{CuO}$ in Fig. 2c shows that tens of CuO NWs construct a yucca fern shaped cluster, which induce a micron-sized porous superficial structure on $\mathrm{Cu}$ foam. The diameter of a single $\mathrm{CuO}$ wire is about $310 \mathrm{~nm}$ as shown in Fig. 3a. A further testing of Fig. $3 \mathrm{~b}$ and c reveals a porous feature of the $\mathrm{CuO} N W$ s. The pore size ranges from 2 to $10 \mathrm{~nm}$. Figure $3 \mathrm{~d}$ presents a high resolution TEM image of a $\mathrm{CuO}$ nanowire. The measured lattice spacing is $0.251 \mathrm{~nm}$, which corresponds to the $(-111)$ lattice plane of monoclinic $\mathrm{CuO}^{22}$. The SAED pattern in Fig. 3e demonstrates that the $\mathrm{CuO}$ NWs are highly crystalline. Finally, a 3D hierarchical porous $\mathrm{CuO}$ structure, containing $\mathrm{Cu}$ foam framework in a few hundred micrometer scale, $\mathrm{CuO}$ wire cluster in a micron-size scale, as well as $\mathrm{CuO}$ NWs with mesopores and micropores, has been achieved.

The above 3D yucca fern shaped $\mathrm{CuO}$ structure is deliberately designed for remitting volume expansion during lithiation/delithiation process, but it is not easy to obtain. Although anodization is not a new technology to fabricate nanowires ${ }^{21}$, it is firstly reported in this study that such method can be used in the preparation of radial $\mathrm{CuO}$ nanowires in strict technological conditions. Figure S2 presents superficial morphologies of $\mathrm{CuO} @ \mathrm{Cu}$ foam anodes in different processing variables. It was found that electrolyte concentration and anodization time only change the size of the obtained nanowires, while temperature and current density are more likely to control the morphologies of the anodized products. By controlling a moderate growth and leaving proper room among adjacent nanowires, yucca fern shaped $\mathrm{CuO}$ structure with plentiful interspaces was obtained successfully.

The crystal structure of $\mathrm{CuO}$ was further investigated by XRD as shown in Fig. 4a. The main diffraction peaks located at $43.3^{\circ}$ and $50.4^{\circ}$ can be indexed to the (111) and (200) planes of Cu phase (JCPDS file No. 04-0836). After the anoidzation and calcination, the diffraction peaks positioned at $35.5^{\circ}, 35.6^{\circ}, 38.8^{\circ}$ and $39.1^{\circ}$ can be assigned to the (002), (-111), (111) and (200) planes of $\mathrm{CuO}$ phase (JCPDS file No. 65-2309), respectively. The EDS results also presented that only $\mathrm{Cu}$ and $\mathrm{O}$ elements exist, as shown in Fig. S3. These results indicate that the $\mathrm{CuO}$, without any other impurities, has been successfully obtained. The porous feature of the 3D hierarchical $\mathrm{CuO}$ NWs was studied by $\mathrm{N}_{2}$ adsorption-desorption measurements as displayed in Fig. $4 \mathrm{~b}$. The pore size ranges from 2 to $20 \mathrm{~nm}$ in the CuO NWs@Cu foam composites. Meanwhile, there are no obvious nanopores on the Cu foam substrate. The isotherm of the composites also exhibits a broad hysteresis as displayed inset of Fig. $4 \mathrm{~b}$, which is also the characteristic of porous structures ${ }^{23}$. The nanopores might greatly improve the electrolyte permeation and provide space for volume expansions during battery cycling. To further gain the surface information on the electronic state of the $\mathrm{CuONWs}$, the XPS analysis was performed as shown in Fig. 4c and d. For the bare Cu foam, the $\mathrm{Cu} 2 \mathrm{p}$ spectrum presents two sharp peaks located at 932.8 and $952.7 \mathrm{eV}$ which are assigned to $\mathrm{Cu} 2 \mathrm{p}_{3 / 2}$ and $\mathrm{Cu} 2 \mathrm{p}_{1 / 2}$, respectively. On the other hand, the $\mathrm{Cu} 2 \mathrm{p}_{3 / 2}$ and $\mathrm{Cu} 2 \mathrm{p}_{1 / 2}$ peaks of $\mathrm{Cu} 2 \mathrm{p}$ electrons for the CuO NWs@ $\mathrm{Cu}$ foam anode shift to the higher binding energies of 934.2 and $954.3 \mathrm{eV}$, respectively. Strong $\mathrm{Cu}^{2+}$ satellite peaks located at $941.8,944.3$ and $962.8 \mathrm{eV}$ are observed in the $\mathrm{Cu} 2 \mathrm{p}$ spectrum of the CuO NWs@Cu foam electrode, confirming the presence of $\mathrm{CuO}$ on the anode surface ${ }^{15}$. The core-level spectrum for $\mathrm{O} 1 \mathrm{~s}$ is displayed in Fig. $4 \mathrm{~d}$. The main peak of $\mathrm{O} 1 \mathrm{~s}$ observed at $529.9 \mathrm{eV}$ corresponds to the binding energy value of $\mathrm{Cu}-\mathrm{O}$, which is a further evidence of the presence of $\mathrm{CuO}$ on the surface of $\mathrm{Cu}$ foam. Other two peaks at 531.6 and $533 \mathrm{eV}$ observed with 

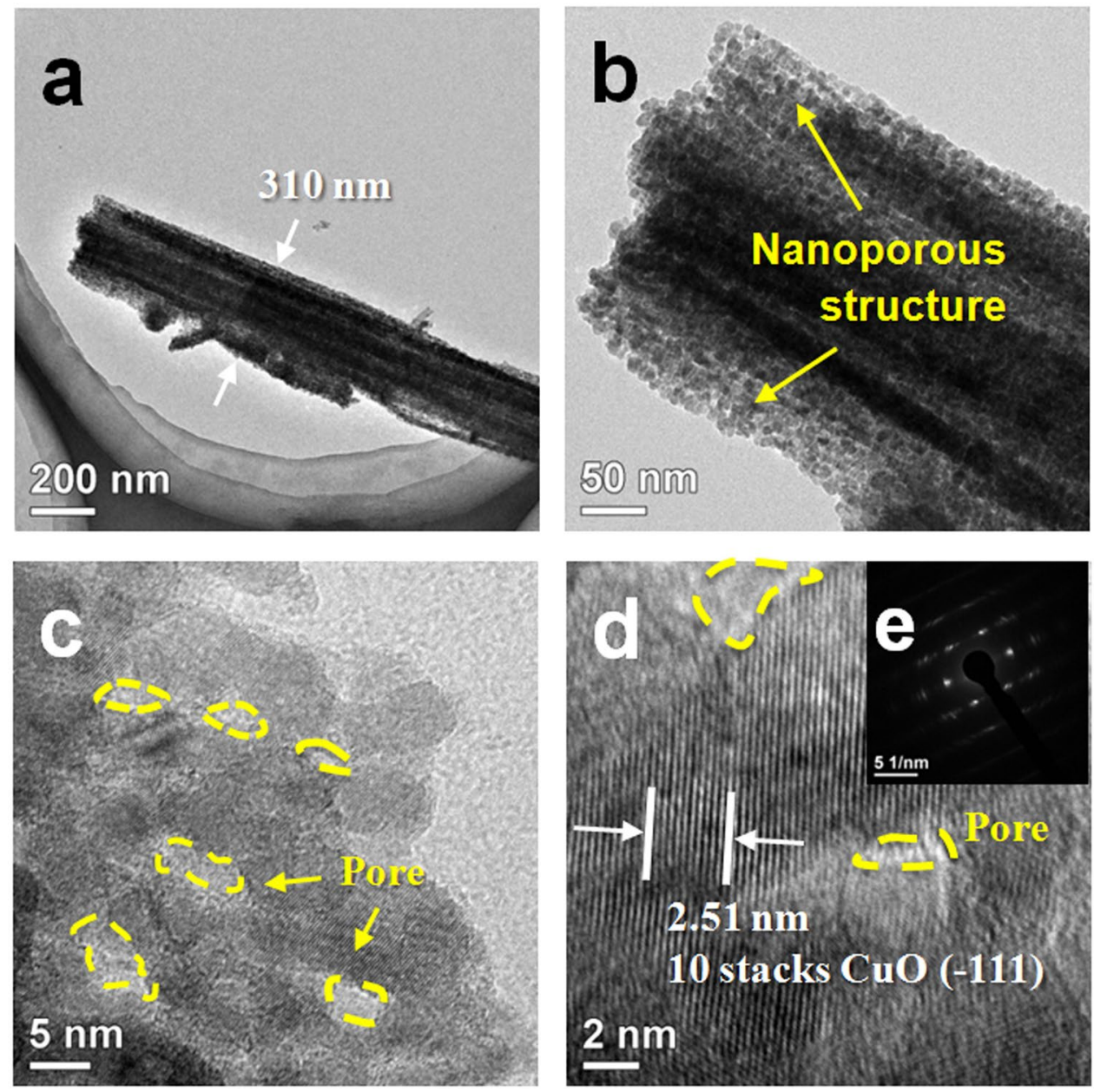

Figure 3. TEM images of porous $\mathrm{CuO}$ NWs at different magnifications (a-d), (e) SAED pattern of the porous $\mathrm{CuO}$ NWs.

higher binding energy values are associated to $\mathrm{OH}$ groups and to water absorbed onto the surface of the $\mathrm{CuO}$ $\mathrm{NWs}$, respectively ${ }^{24}$. For the bare $\mathrm{Cu}$ foam, only peaks at 531.7 and $533.5 \mathrm{eV}$, which may originate from oxygen and moisture in air adsorbed on the $\mathrm{Cu}$ foam surface ${ }^{25}$, have been observed. Thus, possible existence of $\mathrm{CuO}$ on the $\mathrm{Cu}$ foam surface can be ruled out.

The Li ions storage properties of the Cu NWs@Cu foam are carefully evaluated in coin-type cells. The cyclic voltammetry $(\mathrm{CV})$ curves conducted between 0.01 and $3.0 \mathrm{~V}$ at a scan rate of $0.1 \mathrm{mV} / \mathrm{s}$ are displayed in Fig. $5 \mathrm{a}$. It clearly exhibits that three electrochemical reduction steps accompany the lithiation process. During the first cathodic sweep, a broad but weak peak appears at about $2.1 \mathrm{~V}$ which belongs to the initial formation of $\mathrm{Li}_{x} \mathrm{CuO}^{26}$. Then, two succession reduction peaks are observed at $1.0 \mathrm{~V}$ and $0.7 \mathrm{~V}$, which are associated with the further conversion reaction with deep lithiation. A solid electrolyte interphase (SEI) layer formed during the initial discharge process which results in the broaden reduction peaks. During the de-lithiation process, two main peaks at $1.5 \mathrm{~V}$ and $2.5 \mathrm{~V}$, and a shoulder peak at $2.7 \mathrm{~V}$ can be observed, which corresponds to the oxidation of $\mathrm{Cu}$ to $\mathrm{Cu}_{2} \mathrm{O}$ and $\mathrm{CuO}$. In the subsequent $\mathrm{CV}$ cycles, the reduction peaks show a positive shift and demonstrate super reproducibility, indicating the improved kinetics and good reversibility.

The representative galvanostatic discharge/charge curves of the as-fabricated CuO NWs@Cu foam anode are shown in Fig. 5b. There are three plateaus during the initial discharge process, which mainly involve the continuous formation of $\mathrm{LiCu}_{x} \mathrm{O}$ and $\mathrm{Cu}_{2} \mathrm{O}$ phase, as well as the completely conversion to $\mathrm{Li}_{2} \mathrm{O}$ and $\mathrm{Cu}$, respectively ${ }^{17}$. It is also consistent with the results of CV curves. The first discharge and charge capacities are 996.2 and $719.3 \mathrm{mAh} / \mathrm{g}$, which gives the initial Coulombic efficiency of $72.2 \%$. The irreversible discharge capacity is associated with the formation of SEI layer. Two charging plateaus at $2.3 \mathrm{~V}$ and $2.6 \mathrm{~V}$ are observed during the first charging process. In the subsequent cycles, it shows well cycling reversibility and almost similar discharge/charge capacities at the same cycle. The calculated Coulombic efficiency remains $99.57 \%$ last to 100 cycles (Fig. 6a), which indicates the super reversibility. As shown in Fig. 6a, the cycle performance of CuO NWs@Cu foam and CuO NWs@Cu foil substrates were carefully compared. The CuO NWs@Cu foam electrode displayed good cyclic stability and a discharge capacity of $461.5 \mathrm{mAh} / \mathrm{g}$ even at the 100th cycle. In contrast, the CuO NWs@Cu foil electrode gave similar capacities during the initial 10 cycles. However, there is a gradually capacity fading in the following cycles. The capacity retention is only $19.5 \%$ at the 100th cycle for the CuO NWs@Cu foil. This comparison results indicate 

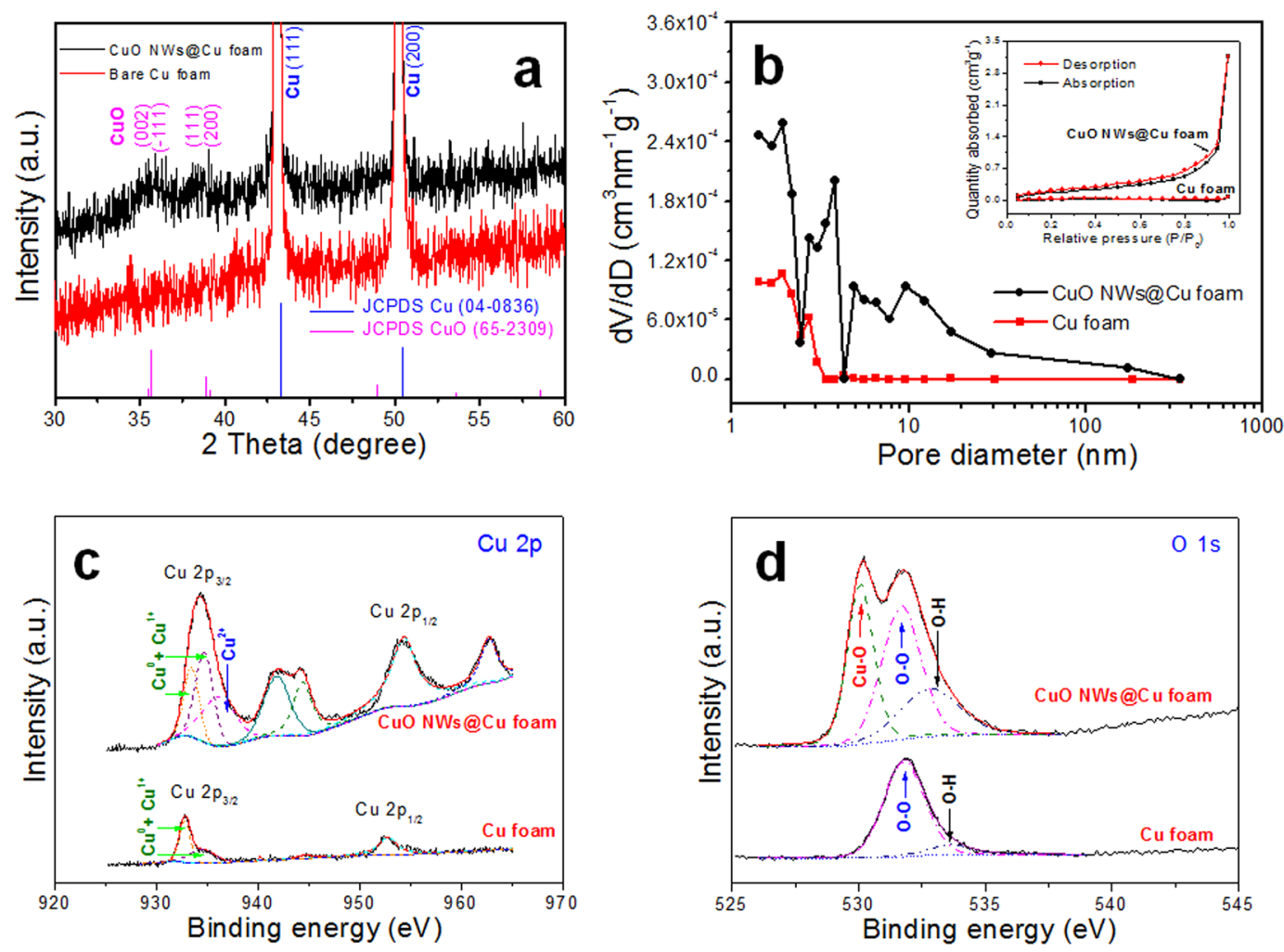

Figure 4. (a) XRD patterns of bare $\mathrm{Cu}$ foam and $\mathrm{CuO}$ NWs@Cu foam, $(\mathbf{b}) \mathrm{N}_{2}$ adsorption desorption isotherms and the corresponding BJH pore-size distribution curves (insets) of $\mathrm{CuO} \mathrm{NWs@Cu} \mathrm{foam,} \mathrm{(c)} \mathrm{Cu} \mathrm{2p} \mathrm{and} \mathrm{(d)} \mathrm{O}$ 1s XPS spectra of bare Cu foam and CuO NWs@Cu foam.

that the 3D structure can be better accommodation for the electrode volume expansions during discharge/charge cycles. The rate capability of the CuO NWs@Cu foam was further investigated at varied current densities (Fig. 6b). The first charge capacities are 792.6, 678.7, 532.2, 315.5 and $150.6 \mathrm{mAh} / \mathrm{g}$ at the current densities of 50, 100, 200, 500 and $1000 \mathrm{~mA} / \mathrm{g}$, respectively. There are two reasons for the excellent rate performance: firstly, the 3D structure features improve the electrolyte permeation and accommodate the volume expansion; secondly, the $\mathrm{Cu}$ foam substrate and the $\mathrm{Cu}$ components at the final discharge state obviously enhance the conductivity of the electrode composite. When the current density returned back to $50 \mathrm{~mA} / \mathrm{g}, 77.9 \%$ of the initial charge capacity was regained. The slight performance fading was due to the formation of stable SEI interface layers. In order to clearly evaluate the performance of CuONWs@Cu foam electrode, a detailed comparison between the present electrode and the reported $\mathrm{CuO}$ electrodes based on different synthesis methods and structures is shown in Table $1^{15,17,25,27-32}$. The superiority of the freestanding hierarchical porous structure of the present electrode, which guaranteed excellent electrochemical properties, can be seen from the table.

To further understand the performance improvement from the $\mathrm{Cu}$ foil to the $3 \mathrm{D} \mathrm{Cu}$ foam substrates, the electrode morphologies after discharge/charge 110 cycles were investigated by SEM as depicted in Fig. 7 . The cycled samples were first disassembled in Ar-filled glove box and washed by electrolyte-free solvent. The surface of the $\mathrm{CuO}$ nanowires were coated by a solid layer and the surface became rough for both of the samples. This rough layer is mainly originated from the decomposition of the electrolyte during the initial discharge cycles. Obviously, some of the thick wires intertwined and merged together to form squid-shaped lumps, which resulted in the performance fading. It is much severe with a $\mathrm{Cu}$ foil substrate because of the inter-cross original architectural feature of the $\mathrm{CuO}$ NWs as shown in Fig. S4. It is nice to see that the $3 \mathrm{D}$ foam architecture and the yucca fern shaped $\mathrm{CuO}$ cluster alleviated this fading tendency and the morphology maintained better, although the nanowire seems to be expanded to some extents. The expansion is due to the unavoidable volume change of transition metal oxides during lithiation/delithiation process as well as the protective effects of stable SEI films. The nanowire grows up a little at every repeated cycle so that it cannot recover to its initial state. The accumulative effect of 110 repeated cycles finally leads to this clear expansion. A further observation on the macro-photograph of CuO NWs@Cu foam anode before and after 110 cycles is shown in Fig. S5. It revealed that there is a good binding force between $\mathrm{CuO}$ active material and the $\mathrm{Cu}$ foam substrate. Barely cracking-off problem is observed for the CuO NWs@Cu foam anode. These results are consistent with the electrochemical performance.

The internal resistance evolutions of the two samples with different $\mathrm{Cu}$ current collectors were also analyzed by electrochemical impedance spectrum (EIS). As shown in Fig. S6, the Nyquist plots are composed of a semicircle followed slope lines, which correspond to the charge-transfer resistance and the Warburg behaviors. It can be seen that two kinds of fresh electrodes exhibit almost the similar resistance (Fig. S6a). After 110 repeated cycles (Fig. S6b), both the resistance reduces to a certain extent as compared to the fresh ones. It can be found 

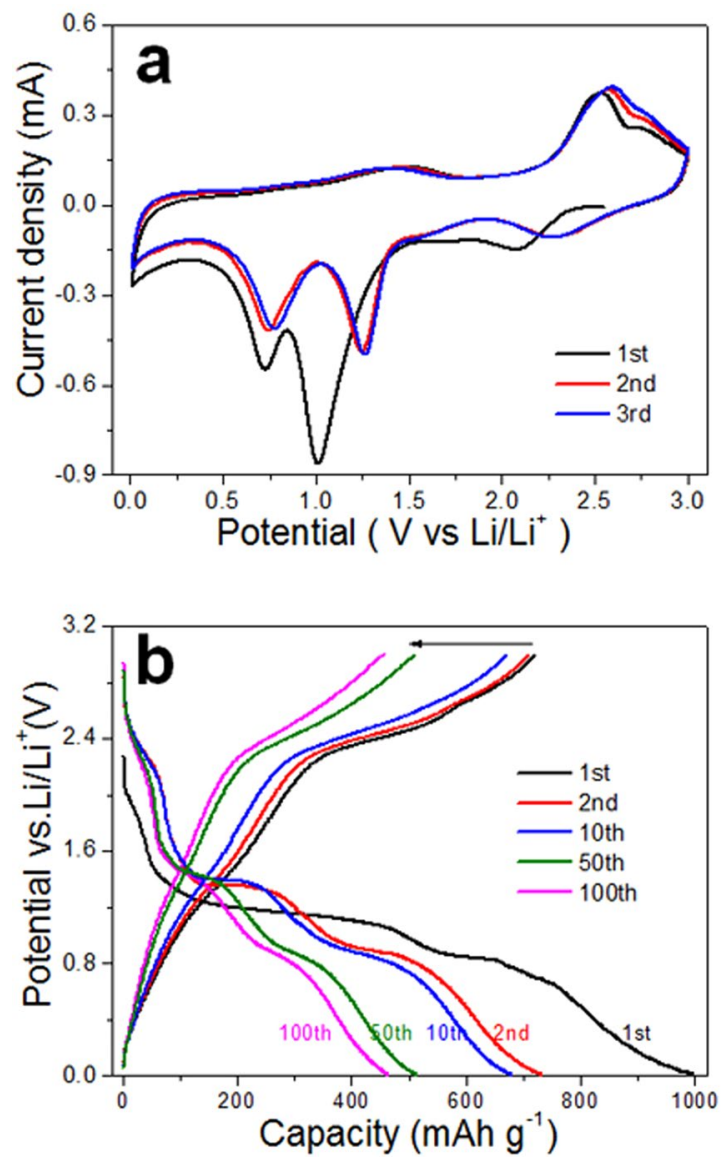

Figure 5. (a) CV curves of CuO NWs@Cu foam at a scan rate of $0.1 \mathrm{mVs}^{-1}$ in the potential range from 0.01 to $3.0 \mathrm{~V}$ vs Li/ $/ \mathrm{Li}^{+}$, (b) Charge-discharge curves of CuO NWs@Cu foam at a current density of $100 \mathrm{mAg}^{-1}$.

obviously that after cycles, the resistance of the electrode with $\mathrm{Cu}$ foam current collector is much lower than that of electrode with $\mathrm{Cu}$ foil current collector. The 3D Cu foam substrate dramatically reduced the resistance, which indicates the better electrochemical kinetics of the Cu NWs@Cu foam than that of CuO NWs@Cu foil.

\section{Discussion}

Formation mechanism of Yucca fern shaped $\mathrm{CuO}$. The structure of $\mathrm{CuO}$ was mainly inherited from Yucca fern structural $\mathrm{Cu}(\mathrm{OH})_{2}$ framework. So, the study on the formation mechanism of the Yucca fern shaped $\mathrm{CuO}$ is indeed an investigation on how $\mathrm{Cu}(\mathrm{OH})_{2}$ framework created.

Every Yucca fern structural $\mathrm{Cu}(\mathrm{OH})_{2}$ cluster is comprised by tens of nanowires. The microscopic formation mechanism of the nanowire is analyzed as follows. The nucleus shape of monoclinic $\mathrm{Cu}(\mathrm{OH})_{2}$ crystal is usually a rod-like structure containing a tip. Under a moderate anodizing condition, most of $\mathrm{Cu}$ ions were transported to tips, resulting in a quick growth of crystals in vertical direction. Only a small part of crystals grow in diameter direction at the same time, so that the growth rate of crystals in vertical direction obviously higher than that in diameter direction. As a result, it is easy to form a wire-like structure under current conditions. If the experimental conditions are changed, the growth rate of crystals in different growth directions may change too. On this occasion, $\mathrm{Cu}(\mathrm{OH})_{2}$ crystals with possible shape of nanoplate and nanosphere instead of nanowire may be generated.

The macroscopic formation mechanism of Yucca fern structural $\mathrm{Cu}(\mathrm{OH})_{2}$ is analyzed as follows. Under suitable anodizing conditions, partial $\mathrm{Cu}$ atoms at the $\mathrm{Cu}$ foam surface were oxidized to $\mathrm{Cu}^{2+}$ ions and immediately reacted with $\mathrm{OH}^{-}$from the alkaline solution to form $\mathrm{Cu}(\mathrm{OH})_{2}$ crystals and deposited on the surface of $\mathrm{Cu}$ foam. With the increase of the anodizing time, $\mathrm{Cu}(\mathrm{OH})_{2}$ kept growing in the solution and formed short nanorods. $\mathrm{Cu}(\mathrm{OH})_{2}$ nanowires were then generated by further growing of these nanorods. Controlling the number of nucleation sites through adjusting anodizing parameters, $\mathrm{Cu}(\mathrm{OH})_{2}$ nanowires were designed to nucleate and grow only at limited sites. Generally, a nucleation site develops into more than one nanowire. Similar to the human hair follicles, one nucleation site is easy to grow into a cluster of radial nanowires, which is named Yucca fern structural nanowires in this study. It needs to be emphasized that the anodizing parameters have an important influence on the formation of Yucca fern structural nanowires. Other morphologies of $\mathrm{Cu}(\mathrm{OH})_{2}$ such as nanoplates, nanowire arrays instead of Yucca fern shape may be prepared by adopting improper experimental conditions. 

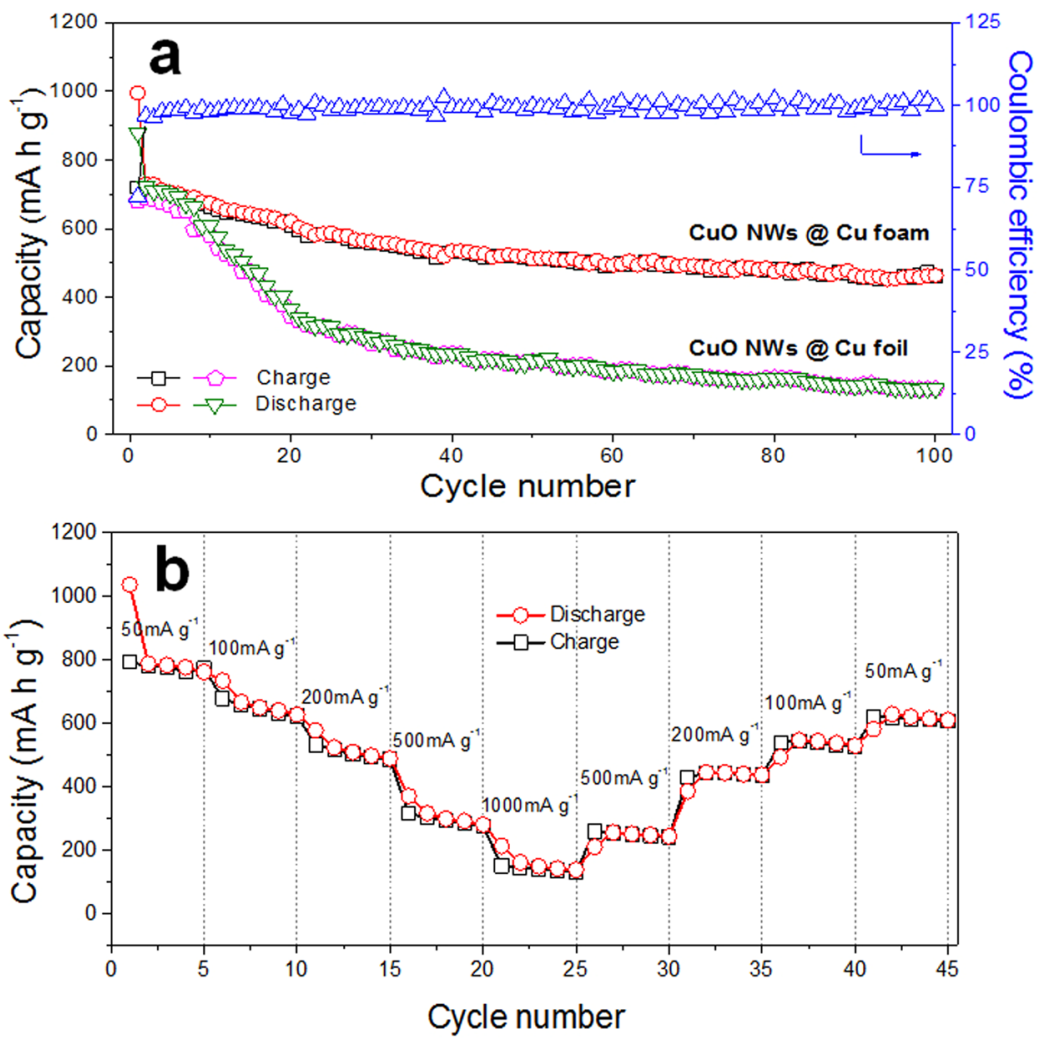

Figure 6. (a) Cycling performance comparison of CuO NWs@Cu foam and CuO NWs@Cu foil at $100 \mathrm{mAg}^{-1}$, (b) Rate capability of CuO NWs@Cu foam electrode at different current densities from 50 to $1000 \mathrm{mAg}^{-1}$.

a

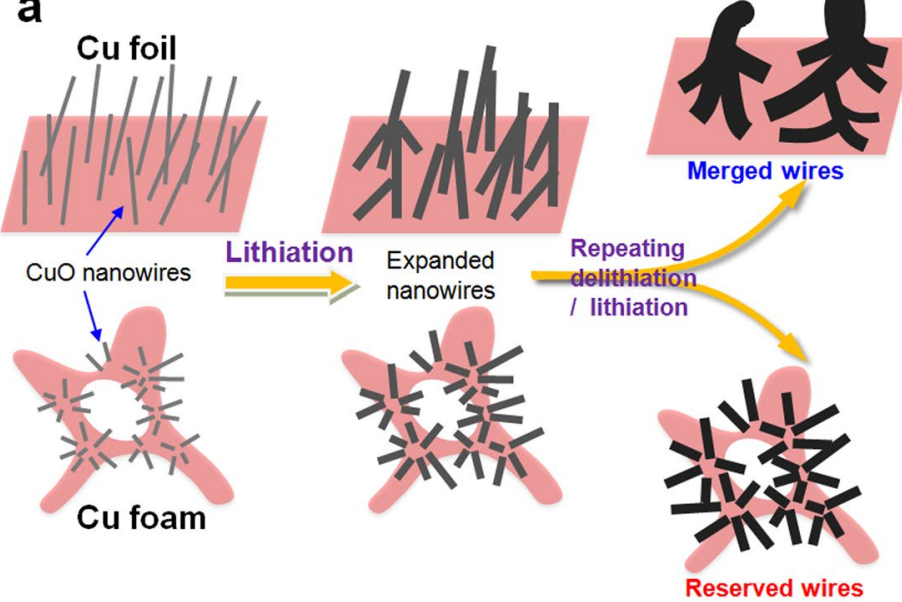

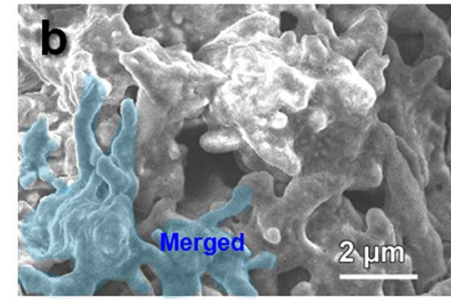

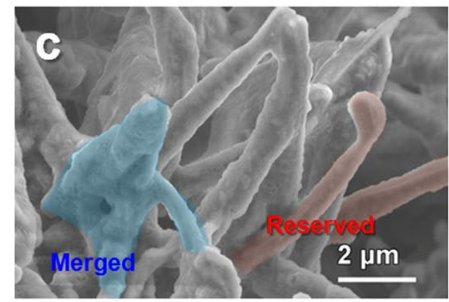

Figure 7. (a) Schematic comparison of the morphological and volumetric changes of CuO NWs@Cu foil and CuO NWs@Cu foam, (b) SEM image of CuO NWs@Cu foil after 110 charge-discharge cycles, (c) SEM image of CuONWs@Cu foam after 110 charge-discharge cycles.

Importance of Yucca fern structure. The hierarchical CuO NWs with Yucca fern structure contains many advantages. Firstly, the 3D structure features can improve the electrolyte permeation and accommodate the volume expansion, which guarantee a good cycling stability. Secondly, the $\mathrm{Cu}$ foam substrate and the $\mathrm{Cu}$ components at the final discharge state greatly enhance the conductivity of the electrode composite, resulting in a good rate property. Based on the results of this work, the relatively stable Yucca fern structure before and after cycling is the key to maintain capacity stability, indicating the significance of this structure in restraining the capacity fading. In addition, in terms of preparation technology, the anodizing is a simple and rapid process to prepare low-cost anodes, which may facilitate the assembly line production. 


\begin{tabular}{|l|l|l|l|l|l|}
\hline CuO materials & Synthesis method & $\begin{array}{l}\text { Current density } \\
\left(\mathbf{m} \mathbf{A ~ g}^{-1}\right)\end{array}$ & $\begin{array}{l}\text { Cycle } \\
\text { number }\end{array}$ & $\begin{array}{l}\text { Capacity } \\
\left(\mathbf{m A ~}^{-1}\right)\end{array}$ & Ref. \\
\hline Leaf-like & Hydrothermal method & 67 & 55 & 421 & 25 \\
\hline Flower-like & Hydrothermal method & 67 & 50 & 392.4 & 17 \\
\hline Octahedral crystals & Reduction and calcination & 134 & 50 & 440 & 26 \\
\hline Nanoplates & Hydrothermal method & 335 & 50 & 281.6 & 15 \\
\hline Dandelion-like & Hydrothermal and calcination & 67 & 50 & 400 & 27 \\
\hline Hollow nanospheres & $\mathrm{Cu}_{2}$ O oxidation & 150 & 50 & 91 & 28 \\
\hline Spherical & Pyrolysis of MOF & 100 & 40 & 500 & 29 \\
\hline Core-shell nanowires & Hydrothermal and oxidation & 100 & 50 & 345 & 30 \\
\hline Bowknot-like & Solution route and calcination & 100 & 30 & 470 & 31 \\
\hline CuO NWs@Cu foam & Anodization and calcination & 100 & 50 & 510.1 & This work \\
\hline CuO NWs@Cu foam & Anodization and calcination & 100 & 100 & 461.5 & This work \\
\hline
\end{tabular}

Table 1. Comparison of capacities of the present work with previous $\mathrm{CuO}$ electrodes reported in the literatures.

If the structure cannot be remained, there must be a clear capacity fading. After 110 repeated cycles, a small part of nanowires had merged together and transformed into squid shape. So, we cannot rule out the possibility of a capacity fading even by utilizing the as-obtained Yucca fern structure during next hundreds of cycles. Anyway, the material has shown excellent performances compared to the existing reports (Table 1). In future work, graphene and other carbon materials were planned to coat on the surface of Yucca fern structural $\mathrm{CuO}$ for improving the long-term stability in both the structure and capacity.

\section{Conclusions}

In summary, a facile and scalable approach for the fabrication of freestanding 3D yucca fern shaped $\mathrm{CuO}$ nanowires with hierarchical porous structure has been introduced. The possible Cu source and substrates have been investigated. The obtained CuO NWs@Cu foam electrode demonstrated superior electrochemical properties, including stable cycle performance $(461.5 \mathrm{mAh} / \mathrm{g}$ after 100 cycles) and high rate capability $(150.6 \mathrm{mAh} / \mathrm{g}$ at current density of $1000 \mathrm{~mA} / \mathrm{g}$ ). The outstanding electrochemical could be attributed to hierarchical porous structured nanowires combined a 3D foam substrate. This unique structure feature guaranteed this Cu NWs@Cu foam a promising anode with a high performance for lithium ion batteries. In addition, the simple fabrication process involving mature anodization technology boosts prospects for scalable production.

\section{Methods}

Materials preparation. The bare $\mathrm{Cu}$ foam with thickness of $0.8 \mathrm{~mm}$ was purchased from Kunshan Jiayisheng Electronics Co. Ltd., China. The anodization was carried out by DC Power Supply (TPR-12010D). Cu foam $(2.0 \mathrm{~cm} \times 4.0 \mathrm{~cm})$ was successively washed with acetone, ethanol, $0.5 \mathrm{M} \mathrm{HCl}$, and deionized water for $10 \mathrm{~min}$ under ultrasonic condition to remove organic contaminants and an oxide layer on surface. And then it was dried using a stream of a compressed air and before using as the working electrode. Another piece of $\mathrm{Cu}$ foam with a same size was selected as the counter electrode. The electrolyte was $1.0 \mathrm{M} \mathrm{KOH}$ solution, which was deaerated by bubbling with dry argon for at least $20 \mathrm{~min}$ before experimentation. The $\mathrm{Cu}$ foam was electrochemically anodized at a constant current density of $10 \mathrm{~mA} \mathrm{~cm}^{-2}$ with a typical reaction time of $10 \mathrm{~min}$ to form $\mathrm{Cu}(\mathrm{OH})_{2} \mathrm{NWs}$. A thermostat was used to control cell temperature at $20 \pm 1^{\circ} \mathrm{C}$. After anodization, samples were rinsed twice with deionized water and then carried out a calcination treatment. The calcination temperature was maintained at $185 \pm 2{ }^{\circ} \mathrm{C}$ for $1.5 \mathrm{~h}$ to converted $\mathrm{Cu}(\mathrm{OH})_{2}$ to $\mathrm{CuO}$. Finally, a binder free $\mathrm{CuO} \mathrm{NWs@Cu} \mathrm{foam} \mathrm{anode} \mathrm{was} \mathrm{obtained.} \mathrm{The} \mathrm{total}$ weight of an anode is about $55 \mathrm{mg}$, in which the weight of active materials is about $6 \mathrm{mg}$. A CuO NWs@Cu foil anode was also obtained through a same process for comparison. All the chemicals were of analytical grade and used as purchased without further purification.

Materials characterization. The morphologies of the as-prepared $\mathrm{CuO}$ NWs and $\mathrm{Cu}$ substrates were observed by scanning electron microscopy (SEM, Nova nanoSEM 450, FEI) equipped with an X-ray energy dispersive spectroscope (EDS) and transmission electron microscopy (JEM-2100F, JEOL). X-ray diffraction (XRD) patterns were obtained using an X-ray diffractometer (D/Max-2500, Rigaku) with $\mathrm{Cu} \mathrm{K} \alpha$ radiation to confirm crystalline structures. X-ray photoelectron spectroscopy (XPS, Thermo Fisher Scientific) was employed to determine the chemical composition and valence state of the anodized products. Nitrogen adsorption/desorption isotherms were measured at 77.2 K with a physisorption analyzer (Micromeritics ASAP 2020). The specific surface area was calculated using the Brunauer-Emmett-Teller (BET) method. The pore size distribution was obtained by the Barrett-Joyner-Halenda (BJH) method.

Electrochemical testing. Electrochemical performances were examined using coin cells (CR2032) assembled in an argon-filled glove box. The cell was consisted of CuO NWs@Cu foam as a working electrode and Li foil as both counter and reference electrode. The electrodes were separated by a Celgard 2400 separator. The electrolyte is composed of a solution of $1 \mathrm{M} \mathrm{LiPF}_{6}$ in a mixture of ethylene carbonate (EC)/dimethyl carbonate (DMC) $(1: 1, \mathrm{v} / \mathrm{v})$ (Shanghai Xiaoyuan Energy Technology Ltd., China). The cyclic voltammetry (CV) test was carried out on an electrochemical workstation (CHI660B, China) from 0.01 to $3 \mathrm{~V}$. Electrochemical impedance spectroscopy 
(EIS) measurements were performed on electrochemical workstation (Princeton Applied Research, PARSTAT 2273), and the frequency ranged from $10 \mathrm{mHz}$ to $100 \mathrm{kHz}$ with an applied AC signal amplitude of $5 \mathrm{mV}$. The charge-discharge test was measured by using a battery testing system (LAND CT2001A, China) in the voltage range of $0.01-3.0 \mathrm{~V}$ (vs. $\mathrm{Li}^{+} / \mathrm{Li}$ ) under different current densities.

\section{References}

1. Dunn, B., Kamath, H. \& Tarascon, J. M. Electrical energy storage for the grid: A battery of choices. Science 334, 928-935 (2011).

2. Larcher, D. \& Tarascon, J. M. Towards greener and more sustainable batteries for electrical energy storage. Nat. Chem. 7, 19-29 (2015).

3. Mahmood, N., Tang, T. \& Hou, Y. Nanostructured anode materials for lithium ion batteries: Progress, challenge and perspective. Adv. Energy Mater. 6, 1600374 (2016).

4. Kim, H. et al. Metallic anodes for next generation secondary batteries. Chem. Soc. Rev. 42, 9011-9034 (2013).

5. Poizot, P., Laruelle, S., Grugeon, S., Dupont, L. \& Tarascon, J. M. Nano-sized transition-metal oxides as negative-electrode materials for lithium-ion batteries. Nature 407, 496-499 (2000).

6. Wang, Z. et al. $\mathrm{CoFe}_{2} \mathrm{O}_{4}$ nanoplates synthesized by dealloying method as high performance Li-ion battery anodes. Electrochim. Acta. 252, 295-305 (2017).

7. Reddy, M. V., Subba Rao, G. V. \& Chowdari, B. V. R. Metal oxides and oxysalts as anode materials for Li ion batteries. Chem. Rev. 113, 5364-5457 (2013).

8. Goriparti, S. et al. Review on recent progress of nanostructured anode materials for Li-ion batteries. J. Power Sources 257, 421-443 (2014).

9. Liu, Y., Cai, X. \& Shi, W. Free-standing graphene/carbon nanotubes/CuO aerogel paper anode for lithium ion batteries. Mater. Lett. 172, 72-75 (2016).

10. Tang, H. et al. Self-assembly of $\mathrm{Si} /$ honeycomb reduced graphene oxide composite film as a binder-free and flexible anode for Li-ion batteries. J. Mater. Chem. A 2, 5834-5840 (2014).

11. Lu, C., Liu, W., Li, H. \& Tay, B. K. A binder-free CNT network- $\mathrm{MoS}_{2}$ composite as a high performance anode material in lithium ion batteries. Chem. Commun. 50, 3338-3340 (2014).

12. Tan, G. et al. Freestanding three-dimensional core-shell nanoarrays for lithium-ion battery anodes. Nat. Commun. 7, 11774 (2016).

13. Mohapatra, S., Nair, S. V., Santhanagopalan, D. \& Rai, A. K. Nanoplate and mulberry-like porous shape of CuO as anode materials for secondary lithium ion battery. Electrochim. Acta. 206, 217-225 (2016).

14. Cheng, S. et al. In-situ oxidized copper-based hybrid film on carbon cloth as flexible anode for high performance lithium-ion batteries. Electrochim. Acta. 212, 492-499 (2016).

15. Liu, X. et al. Morphology- and facet-controlled synthesis of $\mathrm{CuO}$ micro/nanomaterials and analysis of their lithium ion storage properties. J. Power Sources 312, 199-206 (2016).

16. Shi, L. et al. Carbonate-assisted hydrothermal synthesis of porous hierarchical $\mathrm{Co}_{3} \mathrm{O}_{4} / \mathrm{CuO}$ composites as high capacity anodes for lithium-ion batteries. Electrochim. Acta 197, 23-31 (2016).

17. Wang, C. et al. Controlled synthesis of micro/nanostructured CuO anodes for lithium-ion batteries. Nano Energy 9, 334-344 (2014).

18. Yuan, S. et al. Engraving copper foil to give large-scale binder-free porous $\mathrm{CuO}$ arrays for a high-performance sodium-ion battery anode. Adv. Mater. 26, 2273-2279 (2014).

19. Chen, C. et al. Rapid synthesis of three-dimensional network structure $\mathrm{CuO}$ as binder-free anode for high-rate sodium ion battery. J. Power Sources 320, 20-27 (2016).

20. La, D. D., Park, S. Y., Choi, Y. W. \& Kim, Y. S. Wire-like bundle arrays of copper hydroxide prepared by the electrochemical anodization of Cu foil. B. Korean Chem. Soc. 31, 2283-2288 (2010).

21. Li, Z., Chen, Y., Xin, Y. \& Zhang, Z. Sensitive electrochemical nonenzymatic glucose sensing based on anodized CuO nanowires on three-dimensional porous copper foam. Sci. Rep. 5, 16115 (2015).

22. Chen, K., Xue, D. \& Komarneni, S. Beyond theoretical capacity in Cu-based integrated anode: Insight into the structural evolution of CuO. J. Power Sources 275, 136-143 (2015).

23. Bai, Z., Zhang, Y., Zhang, Y., Guo, C. \& Tang, B. A large-scale, green route to synthesize of leaf-like mesoporous CuO as highperformance anode materials for lithium ion batteries. Electrochim. Acta 159, 29-34 (2015).

24. Zarate, R. A., Hevia, F., Fuentes, S., Fuenzalida, V. M. \& Zúñiga, A. Novel route to synthesize CuO nanoplatelets. J. Solid State Chem. 180, 1464-1469 (2007).

25. Wang, C. et al. Morphology-dependent performance of $\mathrm{CuO}$ anodes via facile and controllable synthesis for lithium-ion batteries. ACS Appl. Mater. Interfaces 6, 1243-1250 (2014).

26. Zhu, Y. et al. Facile fabrication of three-dimensional hierarchical CuO nanostructures with enhanced lithium storage capability. RSC Adv. 5, 68061-68066 (2015).

27. Feng, L. et al. Preparation of octahedral CuO micro/nanocrystals and electrochemical performance as anode for lithium-ion battery. J. Alloy. Compd. 600, 162-167 (2014).

28. Xiang, J. et al. Self-assembled synthesis of hierarchical nanostructured $\mathrm{CuO}$ with various morphologies and their application as anodes for lithium ion batteries. J. Power Sources 195, 313-319 (2010).

29. Park, J. C., Kim, J., Kwon, H. \& Song, H. Gram-scale synthesis of $\mathrm{Cu}_{2} \mathrm{O}$ nanocubes and subsequent oxidation to CuO hollow nanostructures for lithium-ion battery anode materials. Adv. Mater. 21, 803-807 (2009).

30. Banerjee, A., Singh, U., Aravindan, V., Srinivasan, M. \& Ogale, S. Synthesis of CuO nanostructures from Cu-based metal organic framework (MOF-199) for application as anode for Li-ion batteries. Nano Energy 2, 1158-1163 (2013).

31. Zhao, Y. et al. Epitaxial growth of hyperbranched $\mathrm{Cu} / \mathrm{Cu}_{2} \mathrm{O} / \mathrm{CuO}$ core-shell nanowire heterostructures for lithium-ion batteries. Nano Res. 8, 2763-2776 (2015).

32. Wang, F. et al. Controlled synthesis of uniform ultrafine $\mathrm{CuO}$ nanowires as anode material for lithiumion batteries. J. Alloy. Compd. 509, 9798-9803 (2011).

\section{Acknowledgements}

The authors would like to acknowledge the financial support from the National Natural Science Foundation of China (51671077, 21603162), China Postdoctoral Science Foundation (2016M600190) and Natural Science Foundation of Hebei Province, China (E2016202212, E2015202081).

\section{Author Contributions}

Z.F.W. and X.Z.L. designed the experiments and wrote the manuscript. Z.F.W., Y.S.Z. and H.Q.X. prepared the samples, performed the experiments, and analyzed the data. C.L.Q. and W.M.Z. helped in writing the manuscript. All authors reviewed the manuscript. 


\section{Additional Information}

Supplementary information accompanies this paper at https://doi.org/10.1038/s41598-018-24963-2.

Competing Interests: The authors declare no competing interests.

Publisher's note: Springer Nature remains neutral with regard to jurisdictional claims in published maps and institutional affiliations.

(c) (i) Open Access This article is licensed under a Creative Commons Attribution 4.0 International License, which permits use, sharing, adaptation, distribution and reproduction in any medium or format, as long as you give appropriate credit to the original author(s) and the source, provide a link to the Creative Commons license, and indicate if changes were made. The images or other third party material in this article are included in the article's Creative Commons license, unless indicated otherwise in a credit line to the material. If material is not included in the article's Creative Commons license and your intended use is not permitted by statutory regulation or exceeds the permitted use, you will need to obtain permission directly from the copyright holder. To view a copy of this license, visit http://creativecommons.org/licenses/by/4.0/.

(C) The Author(s) 2018 